\title{
Factors affecting chemical heat washing efficiency of oily sludge and Demulsification Mechanism
}

\author{
Yong Yang ${ }^{1}$, Yijun Wang ${ }^{2}$. Zebin $\mathrm{Gao}^{2}$, Long Zhang ${ }^{3}$, Chengtun $\mathrm{Qu}^{3}$, Bo Yang ${ }^{4}$ \\ ${ }^{1}$ Yixing Environmental Protection Industry Development Center Yixing, China \\ ${ }^{2}$ Karamay Sanda testing and analysis Co. LTD Karamay, China \\ ${ }^{3}$ College of Chemistry and Chemical Engineering Xi'an Shiyou University Xi'an, China \\ ${ }^{4}$ Shaanxi Key Laboratory of Environmental Pollution Control Technology and Reservoir Protection of Oilfield, Xi'an, China
}

\begin{abstract}
Oily sludge belongs to the hazardous waste specified in the national hazardous waste list. It will directly or indirectly threaten the ecological environment and human health. This paper introduces the advantages of chemical hot washing of oily sludge. This paper discusses the main factors affecting the treatment effect, Demulsification Mechanism and research progress in the treatment of oily sludge by chemical thermal washing, and puts forward some suggestions on the future research and development direction of oily sludge utilization and disposal.
\end{abstract}

\section{Introduction}

Oilfield oily sludge mainly refers to the oil field, the refining of crude oil processing, the process of oil storage, transportation, use, due to the equipment aging, damage, corrosion or operator improper operation and production accidents, create the heavy oil such as crude oil, refined oil, residue "run, run, drip, leak" as the ground oil and mixed with the soil formation of oil, water and mud mixture. The sludge sand discharged from the production system during normal oilfield production can also be classified as oily sludge. It is one of the main nonrecyclable pollutants and is listed as "national hazardous waste"[1].China's petroleum and petrochemical industry produces an average of 800,000 tons of oily sludge every year, while the world produces 60 million tons every year. Generally, the oil content of oily sludge is $10 \% \sim 50 \%$, the moisture content is $40 \% \sim 90 \%$, and the appearance is brown black viscous. At present, a large amount of oily sludge is often produced in the process of crude oil exploitation at home and abroad. Because of the high water content of oily sludge, it will occupy a large amount of land area. Its composition is complex and changeable, belonging to a number of systems, which are generally composed of water in oil $(\mathrm{W} / \mathrm{O})$, oil in water $(\mathrm{O} / \mathrm{W})$ and suspended solids, and usually exist in colloidal and semisolid states[2]. Due to the high emulsification degree of oily sludge, it has high viscosity, poor fluidity and poor heat transfer; moreover, so it is not easy to denaturate, which makes it difficult for the solid phase to settle completely, and the three phases of oil, water and mud are difficult to separate.

\section{Classification and Harm of Oily Sludge}

According to different sources, oily sludge can be roughly divided into the following three categories[3,4]: landing mud: mainly from drilling operations and crude oil extraction process. During well testing operation, oily sludge containing waste drilling mud and drilling cuttings is produced due to oil spill or blowout due to ground pressure; ground mud is produced during crude oil production due to tubing fracture, blowout, sand spitting from oil well and poor anti-seepage; tank bottom mud mainly comes from oilfield gathering and transportation and oil storage process. It includes the bottom mud of crude oil tanks, settling tanks, sewage tanks and oil separators in transfer stations and combined stations (gathering and transportation stations and sewage stations), as well as oily sludge caused by large amount of crude oil or product oil leakage caused by leakage accidents of gathering and transportation pipelines or storage tanks; three sludge in refinery mainly comes from the oil refining process, commonly known as "three sludge". It includes scum on the surface of flotation cell (air flotation tank) produced by water treatment, bottom sludge of oil-water separation tank (oil separation tank), excess activated sludge from biological aeration tank or sedimentation tank or heat exchanger cleaning sludge, waste oil emulsion, etc. This kind of sludge is generally large in yield, and its composition is complex, difficult to degrade, difficult to settle, difficult to concentrate and difficult to treat.

If the oily sludge produced in the process of oilfield development and production is directly discharged into the environment, it will cause serious pollution and even irrecoverable damage to the surrounding soil, water and 
air. The residual oil in the oily sludge will directly or indirectly enter the soil, affect the physical and chemical properties and functions of the soil, cause the nutrient imbalance in various parts of the soil, inactivate plant enzymes, penetrate plant structure, and cause plants to grow normally [5]. Oily sludge contains benzene series, phenols, anthracene, pyrene and other odorous toxic substances. The toxic and volatile components will volatilize in the atmosphere, causing serious air pollution. People and animals inhale a large amount of dust will cause lung disease. Cycloalkanes, alkanes, aromatics and olefins in oily sludge can be enriched by fish and shellfish, and accumulate in human and animal bodies through food chain and food web, resulting in various human diseases. Aromatic hydrocarbons also have mutagenic, carcinogennic and teratogenic effects [6]. Heavy metals such as chromium, mercury, lead and other heavy metals in oily sludge flow into the farmland through material circulation, which will lead to the reduction of beneficial bacteria in farmland soil, the decline of farmland self-regulation ability, and the change of soil morphology. Toxic and harmful substances such as polychlorinated biphenyls, dioxins and radionuclides in oily sludge may cause coma of human and animals, and even cause death at high concentration. Dioxins also have chemical changes in the environment, producing new substances harmful to the environment and human body. If oily sludge is piled up arbitrarily for a long time, it will produce a large amount of methane and other combustible gases under the action of microorganisms, which will not only pollute the air, but also easily cause fire and explosion.

\section{Chemical Hot Washing Technology of Oily Sludge}

At present, the treatment technology of oily sludge at home and abroad has been developing rapidly [7,8]. The traditional incineration method consumes a lot of energy and causes serious secondary pollution; the solidification method not only does not completely decompose pollutants, but also wastes oil resources; the landfill method will occupy a large amount of land resources, which is easier to pollute soil and groundwater than other methods. The common pyrolysis method also has the problems of high energy consumption, high cost and easy to pollute the atmosphere; the modulation mechanical separation method is only limited to the small-scale oily sludge treatment, and the treatment process will produce noise; the solvent extraction method can not deal with heavy metals; the biological method generally has a long treatment cycle. The chemical heat washing method [9] not only achieves the purpose of recycling resources, but also improves the environment. It has the advantages of simple process, mature technology, low energy consumption, low cost, wide application range and good treatment effect. It is suitable for sludge with high oil content. The oil content of sludge can be washed to less than $1 \%$. For small-scale oily sludge treatment, centrifuge and air flotation can be used for three-phase separation; for large-scale treatment, static sedimentation method can be used.
Chemical heat washing is the earliest and most commonly used oily sludge treatment technology. Chemical heat washing method is also called thermal desorption, which is to dilute the cleaning agent (such as hot alkaline water, demulsifier, flocculant, cleaning agent, washing powder or surfactant, etc.) with water under auxiliary means such as stirring and direct heating. The oily sludge is fully contacted with it and washed repeatedly to change the force between oil, water and silt, so that oily substances such as oil with high viscosity are resolved and demulsified, and then separated from the solid surface [10].After mixing and stirring, there will be obvious interface between oil, water and mud, and three-phase separation can be carried out; or the mixed oily sludge can be separated by high-speed centrifuge, and then the mixed oil-water can be separated by air flotation. The separated water phase and hot solution can be recycled. The separated oil phase is sent into the storage tank for resource recovery after treatment. The remaining sludge is treated by dehydration and then treated for harmless treatment or resource utilization.

At present, some scholars combine heat washing with other technologies, and the treatment effect has been greatly optimized. Ma Jiang ping [11] and others have combined the chemical hot washing method with the solidification method, which not only effectively improves the recovery rate of the crude oil in the oil sludge, but also greatly reduces the pollutant content in the sludge, so that heavy metal ions can be solidified and the possibility of secondary pollution is reduced. Lei Jiang hui [12] and others combined the thermal washing method with the biological method, which not only improved the traditional chemical hot washing into biological hot washing, but also changed the thermal washing agent from ordinary cleaning agent to biological cleaning agent, and also further composted the sludge after biological hot washing, and the treatment effect was further optimized, and the pollutants in the sludge were basically degraded and utilized by microorganisms, which had good environmental benefits.

\section{Factors Affecting the Effect of Chemical Heat Washing}

In order to improve the hot washing efficiency of oily sludge and enhance the hot washing effect, it is necessary to study and optimize the key factors in the hot washing process. The factors affecting the treatment effect generally include: liquid-solid ratio of slurry, type of cleaning agent, temperature, stirring intensity, hot washing time and times.

\subsection{Liquid Solid Ratio}

The hot washing of oily sludge usually needs to dilute the sludge first, so as to facilitate the cleaning agent to wash the petroleum substances, refractory organic substances, toxic and harmful substances and sediment in the solid sludge into the liquid phase. When the liquid-solid ratio is too high, the content of liquid in slurry medium is high, which will lead to the increase of treatment capacity and 
the cost of sewage treatment. When the liquid-solid ratio is too low, the content of solid components in sludge water medium is high, which will lead to insufficient washing of oily sludge, and some organic and toxic substances remain in the solid, resulting in inadequate degradation and poor treatment effect.

Lei Jiang hui [12] found that when the liquid-solid ratio was 5 , the oil content of the residual sludge after cleaning was the lowest, about $6 \%$. When the liquid-solid ratio was increased, the oil content of the remaining sludge had an upward trend. Similarly, Pu Yue qi [13] and others also found that when the liquid-solid ratio is about 4.7 , the washing effect is the best. In general, the washing effect is better when the liquid-solid ratio is between 4-7.

\subsection{Selection of Cleaning Agent}

The key of oily sludge hot washing technology is the selection of cleaning agent. Generally, cleaning agents can be divided into single cleaning agent and compound cleaning agent. Single cleaning agent can also be divided into inorganic salt cleaning agent, organic salt cleaning agent (non-ionic cleaning agent, general anionic cleaning agent) and biological cleaning agent. The selection of cleaning agent should be in line with the principles of high efficiency, environmental protection and economy. According to the different properties of oily sludge, the appropriate cleaning agent should be selected to weaken the force between oil, water and sludge, and promote the separation of oil from sludge.

Li Ying [14] and others found that the same cleaning agent has different treatment effect on different oily sludge. The developed reagent $\mathrm{A}$ is suitable for sludge from sewage tank and landing oily sludge, while compound agent $\mathrm{C}$ is suitable for biochemical sludge on the surface of sludge pool. Wang Qi [15] and others found that among the non-ionic cleaning agents with long-chain structure, alcohols and phenols SP169, amines ae9901, resin ta1031 and dendrimer SD-2 have better cleaning effect, and the deoiling rate is above $68 \%$, which indicates that long-chain structure has advantages in cleaning oily sludge containing polymer. After the optimized cleaning conditions, the deoiling rate can reach $91.12 \%$ with $\mathrm{Na} 2 \mathrm{SiO} 3$ and $85.59 \%$ with el100. Baihe [16] and others developed a cleaning agent formula $(2.0 \%$ sodium dodecyl sulfate $+0.25 \%$ Triton TX-100 $+0.86 \%$ sodium silicate $\mathrm{Na} 2 \mathrm{SiO} 3 \cdot 9 \mathrm{~h} 2 \mathrm{o}+5.0 \%$ n-butanol $+91.89 \%$ deionized water) for treating residual oil in oil-based drilling cuttings with high oil content, and the oil removal rate reached $97.3 \%$.

\subsection{Hot Washing Temperature}

The temperature directly affects the separation of oil, sludge and water. This is because the increase of temperature will intensify the thermal movement of particles, increase the chance of collision between particles, and weaken the surface tension, leading to the occurrence of demulsification, which leads to the weakening of oil film adhesion, that is, the oil viscosity is reduced, the oil quality and sludge adsorption force are weakened, and oil will be desorbed from the surface of sludge; at the same time, The activity of the molecules is enhanced, and it is easy to enter the sludge layer of oil at higher temperature, so that crude oil and sludge can be separated. The oil separated from the sludge also floats on the water surface in the form of oil drops due to the increase of temperature and the density difference from water, and the oil-water interface is clear.

However, when the temperature is too high, the water evaporation will increase, the heat loss will increase, and the cost will increase; at the same time, the cleaning agent will evaporate, which will reduce the growth rate of oil removal rate. Therefore, from the perspective of energy consumption cost, the appropriate temperature should be selected in the washing process.

Wang Yong ping [17] and others found that the removal rate of cleaning agent increased by $28 \%$ from $35{ }^{\circ} \mathrm{C}$ to $60{ }^{\circ} \mathrm{C}$, but only $7 \%$ from $60{ }^{\circ} \mathrm{C}$ to $80{ }^{\circ} \mathrm{C}$. When the temperature is over $60^{\circ} \mathrm{C}$, the effect of oil sludge cleaning does not change significantly, so the temperature should be controlled between $55-60{ }^{\circ} \mathrm{C}$ to obtain the best treatment effect. Ma Jiang ping [11] and others found that when the temperature exceeds $40^{\circ} \mathrm{C}$, the removal rate of oil quality by the cleaning agent begins to decline, and the removal rate at $60{ }^{\circ} \mathrm{C}$ is only about half of that at $20{ }^{\circ} \mathrm{C}$. In general, the washing effect is better when the temperature is between $30-80{ }^{\circ} \mathrm{C}$. For oily sludge with different properties, the optimal treatment temperature is slightly different.

\subsection{Hot Washing Stirring Strength}

In the process of hot washing, the stirring intensity will also directly affect the hot washing effect. If the stirring intensity is not enough, the separation of oil and sludge will not be complete; if the stirring intensity is too high, the oil quality and water will become w/o emulsion, which can achieve half the result with twice the effort. Zhang Yuanyuan [18] and others found that with the increase of stirring speed, the oil content of sludge gradually decreased. When the stirring speed exceeds $30 \mathrm{R} / \mathrm{min}$, the oil content decreases slowly. When the stirring speed reaches $50 \mathrm{R} / \mathrm{min}$, the washing phase emulsifies to become w/o emulsion, and it is difficult to separate the intermediate layer from the washing phase. $30 \mathrm{R} / \mathrm{min}$ was selected for hot washing. Li Yichuan [19] and others found that the oil content of sludge first decreased and then increased with the increase of agitator speed, and reached the minimum value at $200 \mathrm{R} / \mathrm{min}$, and the oil content of sludge was about $2 \%$. According to the different oily sludge, the best stirring intensity is different, which needs to be analyzed and tested.

\subsection{Hot Washing Time}

The length of hot washing time will also directly affect the hot washing effect. If the hot washing time is too short, the sludge will not be fully washed, and the oil quality will remain in the sludge; if the hot washing time is too long, it will strengthen the emulsification phenomenon, and is not conducive to the separation of oil quality and sludge. 
Luo Bing bing [20] and others found that the removal rate of oil quality by cleaning agent reached $80.2 \%$ after hot washing for $30 \mathrm{~min}$. After continuous hot washing, the increase of removal rate slowed down, and the hot washing time of sludge in follow-up experiment was set as 30min. Wang Xiao jun [21] and others washed the oily sludge of Liao he Oilfield which oil content is $35.78 \%$ for $60 \mathrm{~min}$, and the oil content of the original sludge decreased to about $7 \%$. However, with the increase of hot washing time, the emulsification of solution becomes more and more serious. After $120 \mathrm{~min}$, the oil content of the original sludge rises to about $8 \%$. The hot washing time of oily sludge should not be too long, which will not only increase energy consumption, but also weaken the hot washing effect.

\subsection{Hot Washing Times}

The hot washing times have great influence on the hot washing effect of oily sludge with high oil content. In order to better integrate oily sludge and medicament and achieve more complete deoiling, two or more hot washing is adopted. Yu Lan lan [22] and others found that during the first hot washing, the deoiling rate was $82.14 \%$; in the second hot washing, the deoiling rate was $83.91 \%$; with the increase of hot washing times, the deoiling rate was no longer significantly increased. From the point of view of saving energy and time, two times of hot washing is selected.

\subsection{Demulsification Mechanism}

Most of crude oil exists in W/O emulsion. There are three conditions for oil and water to form emulsion [23]: there are two immiscible liquids (i.e. crude oil and water); there are emulsifiers (NATURAL emulsifiers in crude oil: asphaltene, gum, resin, silica, clay, etc.); sufficient mixing is required. In recent years, the content of natural emulsifiers in crude oil has increased significantly. They are adsorbed on the oil-water interface to form a layer of viscoelastic membrane, which causes droplet aggregation and kinetic obstacles, resulting in serious emulsification and more stable crude oil emulsion [24]. For different demulsification methods, the demulsifycation mechanism is also different.

Chemical demulsification is to change the interface of emulsion system from stable to unstable by demulsifier, so as to achieve the purpose of demulsification. Demulsifying properties of demulsifier mainly depend on the hydrophilic / lipophilic ability of demulsifier and the ability of breaking the interfacial film. At present, there are four recognized demulsification mechanisms [25]: phase transfer reverse deformation mechanism: after adding demulsifier into emulsion system, surfactant with opposite type to emulsion can be generated as reverse emulsifier (demulsifier), that is, phase transfer occurs. This kind of demulsifier and hydrophobic emulsifier can form complex, which makes emulsifier lose emulsifying property; the mechanism of collision breaking down the boundary film: under heating or stirring conditions, demulsifier will collide with the boundary film, or adsorb on the interface membrane, or replace part of the surface active substance on the interface film, so as to break the boundary film, reduce its stability, and then achieve the demulsification effect through flocculation and coalescence; solubilization mechanism: some molecules of demulsifier form high molecular coil or micelle, which can increase the solubility of emulsifier and cause demulsification of emulsified crude oil; folding deformation mechanism: water in oil emulsion has multilayer water circles, and there are oil circles between water circles. Under the action of heating, stirring and demulsifier, the water circles in the droplets are connected with each other, which makes the droplets agglomerate and demulsify.

Ultrasonic demulsification [26,27] is a new demulsification method. When a certain intensity of ultrasonic wave propagates in emulsion, a series of mechanical, thermal and optical effects will be produced. The main mechanism of ultrasonic demulsification is as follows. Mechanical action: the ultrasonic wave will cause the suspension water and the crude oil medium to vibrate together. The suspended water of different sizes will collide and bond with each other due to the different relative vibration speed, forming water droplets and settling and separating under the action of gravity; Cavitation: when the ultrasonic wave propagates in the liquid, temporary negative pressure will be formed in some areas, resulting in holes or bubbles. When the ultrasonic pressure is negative half cycle, the liquid is pulled, and the bubble core expands rapidly to several times of the original volume. Then when the ultrasonic pressure is positive half cycle, the bubble suddenly collapses and splits into many small bubbles, forming new cavitation Nuclear. Cavitation can produce local high temperature and local high pressure, and is accompanied by strong shock waves and high-speed micro jets. This huge instantaneous pressure can cause the surface of emulsion suspended in liquid to be damaged rapidly, thus promoting oil and water separation; heat action: when ultrasonic waves propagate in the medium, the vibration energy will be absorbed by the medium into heat energy, which will destroy the stability of the emulsion.

The biological demulsification method made the emulsion demulsifying and dehydrating [28] by adding microbial fermentation medium. It uses the growth and development of microbial cells, metabolism and metabolites to reduce the surface tension of oil-water interface and destroy the stability of emulsion, so as to achieve the purpose of demulsification oil-water separation. Biological demulsification has the advantages of simple process, low cost, low energy consumption and easy commercialization. There are three main ways of demulsification: microbial cell demulsification; biosurfactant demulsification produced in the process of microbial fermentation; and microbial metabolic process demulsification.

Microbial species, age and other factors have an important impact on the demulsification effect, and the screening of bacteria has become one of the important contents of biological demulsification research. 


\section{Conclusion and Prospect}

In order to promote the practical application of chemical heat washing in oily sludge treatment, the following aspects can be strengthened

The research on the interaction mechanism between cleaning agents is less, so it is necessary to study the synergistic effect mechanism between cleaning agents to optimize the detergent formulation;

The results show that there may be synergistic effects among various process intensification methods, and the combination of these enhancement methods is helpful to further improve the oil washing removal effect;

Due to the complexity of oil composition, the properties of oily sludge are complex and changeable, which makes the chemical hot washing process and effect different. Therefore, the influence of oily sludge properties on the chemical hot washing process can be systematically studied to provide basic data for detergent formulation research;

Generally, chemical heat washing process can reduce wastewater discharge by optimizing operation conditions and reusing washing wastewater, but the reuse times of washing wastewater are limited. Therefore, how to effectively treat washing wastewater and reduce the secondary pollution of wastewater is another urgent problem to be solved.

Inorganic pollution in oily sludge only had a single treatment technology which can not meet the requirements of environmental protection, nor can be utilized as resources. Therefore, it is necessary to combine various treatment technologies according to the characteristics of different treatment technologies, so as to expand the scope of use and improve the treatment efficiency. The deep disposal of oily sludge in different grades and stages has become the focus of the development of oily sludge treatment technology.

\section{References}

1. Ministry of environmental protection, national develop-ment and Reform Commission, Ministry of public security. National hazardous waste list [EB / OL] (2016-06-14)

2. Chieng S,Wang Y H,Gao N B,et al.Pyrolysis of oil sludge with oil sludge ash additive employing a stirred tank reactor[J].journal of Analytical and Applied Pyrolysis,2016,120:511-520.

3. Wang Yuhua, Chen chuanshuai, Meng Juan, Han Yunying, Li Aimin, Wang Dong. New development and application status of oily sludge disposal technology $[\mathrm{J}]$. Safety and environmental engineering, 2018,25 (03): 103-110

4. Mrayya B,Battikhi M N,Biodegradation of total organic carbons (TOC) in Jordanian petroleum sludge [J].journal of Hazardous Materials, 2005, 120(1/2/3): 127-134

5. HUI K, TANG J, LU H, et al. Status and prospect of oil recovery from oily sludge: A review[J]. Arabian Journal of Chemistry, 2020
6. HU G, FENG H, HE P, et al. Comparative life-cycle assessment of traditional and emerging oily sludge treatment approaches[J]. Journal of Cleaner Production, 2019

7. MOHIT M A, GHAZBAN F, OMIDVAR B. Optimization of influential parameters of hydrocarbon recovery from waste oily sludge by solvent extraction using solvent blend[J]. 2020

8. VARJANI, SUNITA, J. Microbial degradation of petroleum hydrocarbons[J]. Bioresource Technology Biomass Bioenergy Biowastes Conversion Technologies Biotransformations Production Technologies, 2017

9. Bai Yu, Cheng Yuanpeng, Hu Jiujiang, Huang Chunfeng, Tang shanfa. Research status and Prospect of thermal cleaning technology for oily sludge [J]. Applied chemical industry, 2020,49 (06): 1498-1501 $+1507$

10. Ying L, Xi C, Xiumin L, et al. Engineering Application of Chemical Thermal Washing for Oily Sludge[J]. Environmental Protection of Oil \& Gas Fields, 2018.

11. Ma Jiangping, Zhao Honggang, Wang Feng, et al. Experimental study on oily sludge treatment by chemical hot washing and heavy metal solidification $[\mathrm{J}]$. Environmental protection of oil and gas field, 2015, 025 (003): 18-20

12. Lei Jianghui. Laboratory experimental study on treatment of oily sludge by biological hot washing and microbial degradation technology [J]. Oil and gas field surface engineering, 2020,39 (04): 40-44

13. Pu Yueqi, Yu Chao, Li Zhiliang, et al. Study on rhamnolipid enhanced thermochemical washing of oily sludge [J]. Journal of Zhejiang Ocean University (NATURAL SCIENCE EDITION), 2014 (33): 572575

14. Li Ying, Chen Xi, Li Xiumin, et al. Engineering application of chemical heat treatment of oily sludge [J]. Environmental protection of oil and gas field, 2018, 028 (001): 14-16

15. Wang Qi, Li Meirong, Zhu Wei, et al. Effects of different types of cleaning agents on the cleaning effect and interfacial properties of oily sludge containing polymer $[\mathrm{J}]$. Journal of environmental engineering, 2012

16. Baihe, Wu Weifeng, Weng Liangyu, et al. Study on chemical cleaning treatment of oil-based drilling cuttings with high oil content [J]. Natural gas and petroleum, 2018, 036 (003): 76-80

17. Wang Yongping, Zong Tinggui, Zhang Lei. Study on ultrasonic enhanced hot washing process of oily sludge [J]. Petrochemical application, 2016, 35 (010): 145-148

18. Zhang Yuanyuan, Yu Yin. Study on harmless treatment technology of oily sludge based on chemical washing [J]. Xinjiang environmental protection, 2018, 40 (03): 32-38 
19. Li Yichuan, Wang Dong, Wang Yu, et al. Washing sludge by thermochemical cleaning -- Study on process conditions of oil recovery $[\mathrm{J}]$. Cleaning world, 2008 (11)

20. Luo Bingbing, Huang zuhao. Study on treatment of oily sludge by hot washing process [J]. Yunnan Chemical Engineering, 2018, 45 (08): 75-76

21. Wang Xiaojun, Wu Yue. Screening of washing agents for oily sludge treatment in oil field [J]. Liaoning chemical industry, 2017 (04): 325-328

22. Yu Lanlan, Song Jian, Zheng Kai, et al. Study on hot washing process for oily sludge [J]. Chemical technology, 2014, 22 (001): 29-33

23. .Mu Jianhai. Study on Demulsification Mechanism of crude oil and development of demulsifiers [J]. Chemical technology market, 2002, 25 (004): 26-28

24. Cherney D P, Wu C, Thorman R M, et al. Investigating the Impact of Crude Oil Solubility on Water-in-Oil Emulsion Stability and Its Relation to Molecular Composition of Crude Oil at the OilWater Interface[J]. Energy \& Fuels, 2015, 29(mayjun.):3616-3625.

25. Feng, Jie, Cao,Effect of Demulsifier Structures on the Interfacial Dilational Properties of Oil-Water Films $[\mathrm{J}]$. Journal of Dispersion Science \& Technology, 2016.

26. Li Zhongjie, Tong Zhenzhen. Application status of ultrasonic in petroleum engineering [J]. Contemporary chemical research, 2020 (13): 7-8

27. Wang Yongping, Zong Tinggui, Zhang Lei. Study on ultrasonic enhanced hot washing process of oily sludge [J]. Petrochemical application, 2016,35 (10): $145-148+153$

28. Cai Q, Zhu Z, Chen B, et al. Oil-in-water emulsion breaking marine bacteria for demulsifying oily wastewater[J]. Water Research, 2019, 149(FEB.1):29. 